\title{
Design and Realization of Fault Restoration System
}

\author{
WANG Zhi-gang ${ }^{1, a}$, LI qiang $^{1, b}$, LI chunhui ${ }^{1, c}, X^{2}$ Lei $^{1, d}$ \\ ${ }^{1}$ Shanxi Electric power corporation Economic and technical institute of State Grid, Taiyuan, \\ 030001, China
}

awangzhigang@sx.sgcc.com.cn, bvictorlq@163.com, ${ }^{\mathrm{C} l i c h u n h u i @ s x . s g c c . c o m . c n, ~}{ }^{\mathrm{d}}$ hulate@126.co $\mathrm{m}$

Keywords: Fault restoration; Power grid; Subsystem-switch; Black-start.

\begin{abstract}
A regional network fault restoration system that can be applied in on-line mode is researched and realized. According to the power outage, this fault restoration system can recover the electricity rapidly after small scope fault, meanwhile, the system can propose the black-start scheme when blackout occurs. On the basis of switch-subsystem model, proposing the new searching strategy for restoration path to improve the efficiency and accuracy of the search. The proposed fault restoration system has been put into operation in regional power network, the validity of the system has been verified.
\end{abstract}

\section{Introduction}

Fault restoration refers to restore electricity for the out-of-service areas of a system due to the isolation of a fault as soon as possible. With the structure of power grid more and more complex, the scale larger and larger, the requirement of customers for power quality increasing rapidly, the allowed blackout interval getting smaller, it made that the grid can achieve the fault restoration efficiently and effectively. Especially under the smart grid environment, self-healing is a basic characteristic of smart grid. That's what does really matter is how to make restoration put into use[1-4].

As far as right now, on-line restoration system focus on the simple fault in small scope, lack of the consideration for black start in large outages. In the existing literature [5-6], commonly use the network topology to get the node-switch model. Combine with charging tag to search recovery path based on the equivalent model. The method is pragmatic, but the search speed is subject to the number of nodes.

In order to meet the actual needs of the fault recovery, first estimate fault blackout range, determine whether it's necessary to provide the black-start or not. Meanwhile, based on the above node-branch model, the paper adopts the connection analysis to build the subsystem-switch model to search the optimal recovery path.

The fault recovery strategy needs to satisfy several requirements as follows:

- Real-time. It's necessary to restore the electricity as soon as possible, to improve the service reliability, to meet the requirements of the customers.

- Restore the loads as much as possible. The more significant the loads, the higher the recovery priorities.

- Little change in the network structure.

- Avoid the overload during the process of the recovery.

In addition, an attempt of the recovery strategy to minimize the power losses and the number of switching operations is made.

\section{Fundamental Function of Restoration System}

The specific functions which the restoration system realizes are as follows: 
- Using black-start power, supply effective and reasonable paths to restore the power system when blackout occurs.

- Eliminate the line overloads.

- Search all the possible recovery way, evaluate the paths to select the optimal one, form the recovery scheme.

- Format accurate operation orders to provide convenience for the operators.

Have interface with other related systems: obtain the state of circuit breakers, as well as real-time, historical information and telemetering from the SCADA and fault information system; receive results of diagnosis from the diagnosis system; show recovery path dynamically through interactive interface.

\section{Basic Structure of Restoration System}

The fault restoration system consists of external data source, data base, fault restoration module and interactive interface. The external data source obtains the information from the SCADA, fault information system and diagnosis system. The properties and connectivity of the devices are stored in the data base, to provide data service for the fault recovery. Fault restoration is the core module of the system. The module can isolate the faulted devices and provide optimal paths for the unfaulted outage areas. The recovery path can be showed dynamically through interactive interface. The functional diagram of the restoration system module shows as Fig.1.

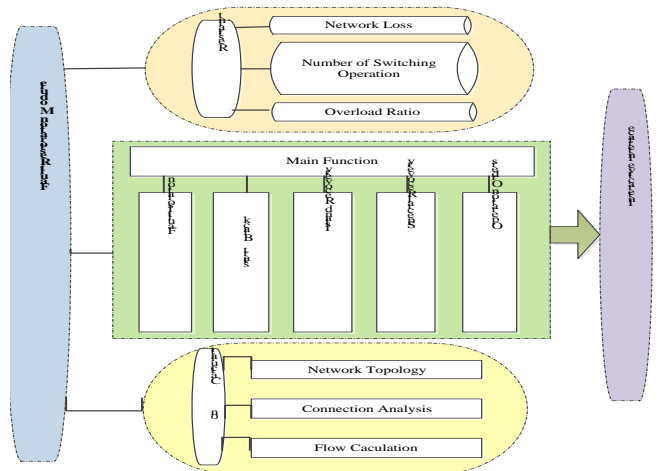

Figure 1. Functional diagram of restoration system module

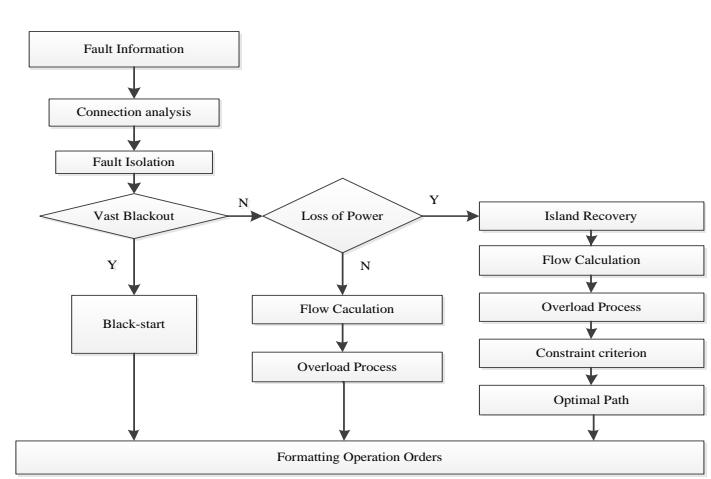

Figure 2. Process of the recovery.

As the Figure1 shows, the fault restoration module consists of the main function module, computation module and the restricting module.

The computation module can realize the network topology, connection analysis and load flow calculation. The restricting module can evaluate all the paths to select the optimal one based on the network loss, the number of switching operations and the overload ratios.

The main function of the restoration module includes fault isolation, black-start, island recovery, special recovery for fault and the forming of the operation tickets. The specific function shows as follows.

Fault Isolation. When obtain the specific diagnosis information from the diagnosis system, it's necessary to isolate the fault devices. When isolate the devices, the switches connected with the fault devices need to be disconnected quickly, to avoid to enlarge the de-energized areas.

Black-start.Black start is one of the important means for quick recovery of electric power system failure, and it can reduce the loss and meet the demand for emergency use. It means that under the circumstances of all black-out, using the ideal black start power, to bring other generators to start up, to restore the entire system gradually $[8,9]$.

Based on the features at different moments of time, the black-start can be divided into three different periods, black start, network reconfiguration and load restoration respectively [10-13]. The key point of the first period is to select ideal black start power, to divide the system to several subsystems reasonably. During the reconfiguration period, take full advantage of the restored 
generating capacity, to bring other generators to start up, to put the transmission line to realize skeleton restoration. It's necessary to restore the load, to incorporate subsystems rapidly during the load restoration period.

Island Recovery.De-energized islands include faulted outage subsystem and unfaulted outage subsystem. The faulted outage subsystem has been isolated by the isolation module, while the unfaulted outage subsystem needs to be restore electricity as soon as possible. This paper provides a recovery strategy based on the subsystem-switch model. The strategy can be discussed in detail in next section.

For the unfaulted outage subsystem, it's necessary to evaluate the network loss, flow overload ratios and the number of switching operations to select the optimal path.

Special Recovery for Fault.The load with special demands as power cut requires special handling, to satisfy the requirements of safe operation of power network.

Operation Tickets.Based on operating rules and actual state of electric equipment of substation, give correct operation order to guarantee for steady performance of substations and power system.

Figure 2 shows the full process of the recovery.

The fault of the power gird can lead to two different results:

- Lead to the de-energized areas;

- Lead to the overload of the devices.

Based on the different results, the recovery tasks can be divided to two types, searching the optimal path for the de-energized areas; searching the reasonable switching path for the overload devices respectively. Which is truly remarkable, it's necessary to present black-start scheme when blackout occurs.

\section{Search Method Based on the Subsystem-switch}

Equivalent Model of the Power Grid. When studying the fault restoration, the network topology analysis is commonly used for the equivalent analysis of power grid. The abstract model of the electrical equipment shows as endpoint-branch [14-15]. Based on the topology analysis, the power grid can be equivalent to the node-branch model. The node means the integration of the endpoints connected by non-impedance branch. Judge the connectivity of the node, combined with the open-close state of the switches. The interconnected nodes are merged to form a subsystem. The subsystem is a collection of nodes, the grid is equivalent to subsystem-switch model.

There are three different kinds of subsystems, including charged subsystem, uncharged subsystem, and black-out subsystem respectively. Uncharged subsystem is the subsystem which is uncharged before and after the fault; black-out subsystem is the subsystem which is charged before the fault, but uncharged after the fault. Black-out subsystem can be divided into the faulted outage subsystem and unfaulted outage subsystem. The faulted outage subsystem contains fault devices, the unfaulted outage subsystem is on the contrary.

In order to ensure the completeness of searching path, another classification of the subsystem is introduced. The subsystem can be divided into the terminal subsystem and non-terminal subsystem. Terminal subsystem is uncharged subsystem, and its adjacent subsystem has a fault flag or search flag. During the search process, while the terminal subsystem is searched, if the adjacent subsystem of terminal subsystem is charged, it may be marked as a recovery way, or the search is complete.

Search Recovery Path Based on the Equivalent Model.In order to meet the actual needs of the fault recovery, using the breadth-first search strategy, combined with the above shown grid equivalent model, a fast search method for power grid fault restoration is presented. 


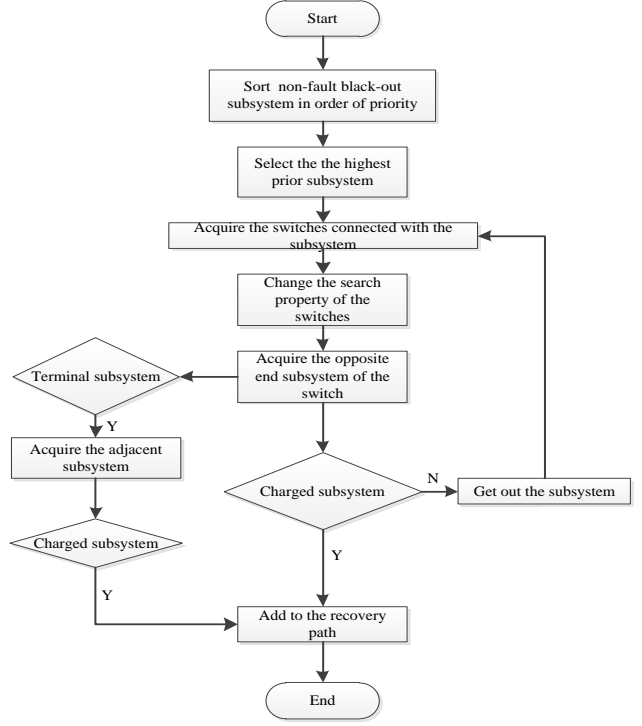

Figure 3. Process of the search method.

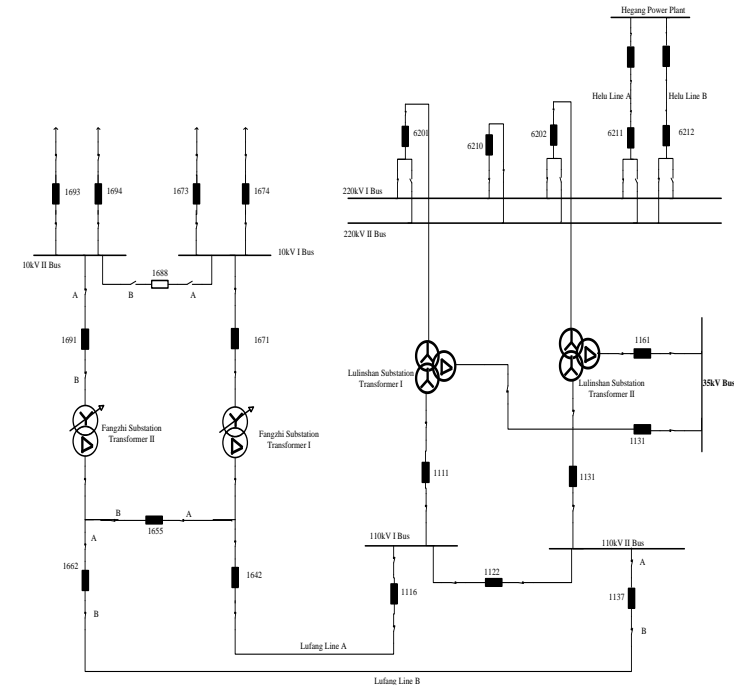

Distribution Network Map

Firstly, sort unfaulted outage subsystem in order of priority in accordance with the degree of importance of the load. Start the search from the highest prior subsystem, find all adjacent subsystems by searching the connected switches, repeating operations until you find the charged or terminal subsystem. Update the property of switch dynamically during the searching process. When searching a switch, the property of the switch will be searched. Figure 3 shows the full process of the search method.

\section{Software Testing}

In this section, the proposed fault restoration system has been put into operation to verify the validity in distribution system of large scale shown in Fig.4 (Black means the circuit breaker is closed, white is on the contrary.)

Under the normal operation, all the buses, lines, the transformers and breakers are in service in Fangzhi and Lulinshan Substation except 10kV bus-tie switch in Fangzhi Substation. After the failure of the transformer II, the connected breakers 1662, 1691 trip. The fault leads to the blackout of the $110 \mathrm{kV}$ Lufang Line B and the 10kV II Bus in Fangzhi Substation. Start the restoration system, isolate the faulted devices, search the recovery path for the de-energized areas, form the operation orders as Fig. 5 shows.

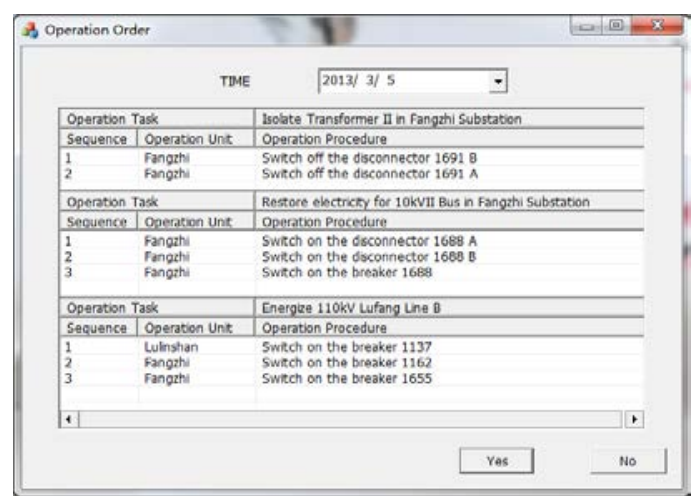

Figure 4. Operation Order

\section{Conclusion}

- According to the power outage, this fault restoration system can recover the electricity rapidly after small scope fault, meanwhile, the system can propose the black-start scheme when blackout 
occurs.

- Provide the subsystem-switch equivalent model of the power grid. Based on the equivalent model, using the breadth first strategy to search the recovery path. Select the optimal path by evaluating the number of switching operations, overload ratios and network loss. The method possesses such advantages as high efficiency and operability.

- Based on the characters of different grids, the special recovery module is installed to deal with the special loads, to satisfy the requirements of safe operation of power network.

\section{References}

[1] Liu Daobing, Gu Xueping and Zhao Jieqiong, "Practical research of fault restoration for the regional power grid,” Power System Protection and Control, Vol. 38, No. 21, 2010, pp. 48-52.

[2] Chao-Ming Huang, Cheng-Tao Hsieh and Yung-Shan Wang, "Evolution of radial basic function neural network for fast restoration of distribution systems with load variations," Electrical Power and Energy Systems, vol. 33, 2011, pp. 961-968.

[3] Cuong P. Nguyen and Alexander J. Flueck, “Agent Based Restoration With Distributed Energy Storage Support in Smart Grids," IEEE Transactions on Smart Grid, vol.3, No.2, 2012, pp. 1029-1038.

[4] Wu Ye, Fang Xin-yan and Zhang Yan, "Tabu search algorithm based black-start zone partitioning,” Power System Protection and Control, Vol. 38, No. 10, 2010, pp. 6-11.

[5] Zhang Haibo, Zhang Xiaoyun and Tao Wenwei, "A breadth-first search based service restoration algorithm for distribution network,” Power System Technology, Vol. 34, No. 7, 2010, pp. 103-108.

[6] Zheng Chaohong, Bie Chaohong and Wang Xiuli, “A Fast Heuristic Service Restoration Method for Distribution Network,” Electric Power Automation Equipment, Vol. 24, No. 2, 2004, pp. 16-19.

[7] Liu Ming-hui, Zhang Dong-ying and Zou Pin-yuan, "Regional Network Fault Restoration System Applied in On-Line Mode,” Power System Technology, Vol. 30, No.18, 2006, pp. 35-39.

[8] Liu Ying-shang, Wu Wen-chuan and Feng Yong-qing, "Black-start zone partitioning based on ordered binary decision diagram method," Proceedings of the CSEE, Vol. 28, No. 10, 2008, pp. 26-31.

[9] Zhou Yun-hai and Min Yong, "Optimal algorithm for system reconstruction," Proceedings of the CSEE, Vol. 23, No. 4, 2003, pp. 67-70.

[10]Luo Ping-ping, Lin Ji-keng and Li Ding, "Search Algorithm of Black Start Initial Scheme," Electric Power, Vol. 44, No. 5, 2011, pp. 19-25.

[11]Liu Qiang, Shi Libao and Ni Yixin, "Intelligent Optimization Strategy of the Power Grid Reconfiguration During Power System Restoration,” Proceeding of the CSEE, Vol. 29, No. 13, 2009, pp. 8-15.

[12]Liu Ying-shang, Wu Wen-chuan and Feng Yong-qing, "Load Recovery during Power System Blackstart,” Power System Technology, Vol. 31, No. 13, 2007, pp. 17-22.

[13]Chen Xiaoping and Gu Xueping, "Determination of the Load Restoration Plans Based on Genetic Simulated Annealing Algorithms,” Transactions of China Electro Technical Society, Vol. 24, No. 1, 2009, pp, 171-176.

[14]Chen Xing-ying, Sun Shun-jian and Qian Feng, “A fast power system network topology based on tracking technology,” Power System Technology, Vol. 28, No. 5, 2004, pp.22-24.

[15] Song Shao-qun, Zhu Yong-li and Yu Hong, “A power network topology tracking method based on graph theory and artificial intelligence search technique,” Power System Technology, Vol. 29, No. 19, 2005, pp. 45-49. 